\title{
Switching pure states of the dissipative Heisenberg $X X Z$ chain by local magnetic fields
}

\author{
Vladislav Popkov ${ }^{1,2}$ and Mario Salerno ${ }^{3}$ \\ ${ }^{1}$ Department of Physics, Bergische Universität Wuppertal, 42097 Wuppertal Germany \\ ${ }^{2}$ Department of Physics, University of Ljubljana, Jadranska 19, SI-1000 Ljubljana, Slovenia \\ ${ }^{3}$ Dipartimento di Fisica "E.R. Caianiello", and Istituto Nazionale di Fisica Nucleare - Gruppo Collegato di Salerno, \\ Universitá di Salerno, Via Giovanni Paolo II, 84084 Fisciano (SA), Italy
}

\begin{abstract}
The effects of a local magnetic field on nonequilibrium stationary states (NESS) of the open quantum $X X Z$ spin chain are investigated with a Lindblad master equation approach in the limit of strong dissipation. The local magnetic field is applied to a single bulk spin of the chain while the ends are kept at fixed polarizations by dissipation. We show that suitable changes of the local magnetic field permit to invert the spin current by switching pure NESS with opposite chiralities, while preserving the state purity and achieving optimal transport.
\end{abstract}

PACS numbers: 75.10.Jm, 75.76.+j, 03.60.-k, 03.67.Pp

Introduction. Much efforts are presently devoted to the study of quantum properties, such as entanglement and purity, that allow to select within the huge Hilbert space of a quantum many-body system, states that are useful for quantum information and for the development of new quantum devices. As it is well known, entangled states are fundamental for quantum protocols involving key distribution, error corrections, teleportation, etc., while pure states play a crucial role for security and efficiency of these protocols [1]. In the real world, however, a quantum system is always in contact with some environment which leads to decoherence and therefore to the loss of the above properties quickly in time. This implies that quantum states of realistic many-body systems are rarely pure.

Recently it has became clear that it is possible to stabilize quantum states by means of dissipation [2-12]. This approach has many practical advantages with respect to the creation of the same states via coherent dynamics, since the quantum states produced by means of dissipation are intrinsically nonequilibrium stationary states (NESS) not reachable within Gibbs ensemble and much more resistant against decoherence.

On the other hand, the management of NESS is far from being trivial and typically requires the use of sophisticated dissipative long range mechanisms [13]. In particular, to change a dissipatively produced pure state into another pure state with qualitatively different physical properties, nontrivial changes of either the coherent part of the dynamics, or of the dissipators, or of both, are required [14]. All these operations involve structural changes that are not easy to implement in real experiments. For practical applications it would be therefore convenient if the management of dissipatively produced states could be realized by means of external fields, without making any structural change to the system. In particular, if two pure NESS carrying opposite currents could be switched under this type of management, this would allow to realize a quantum switch that inverts the current simply by means of a local field.

The purpose of this Letter is to show how such a quantum switch can actually be made. For this we investigate the NESS of an open quantum $X X Z$ spin chain in the limit of strong

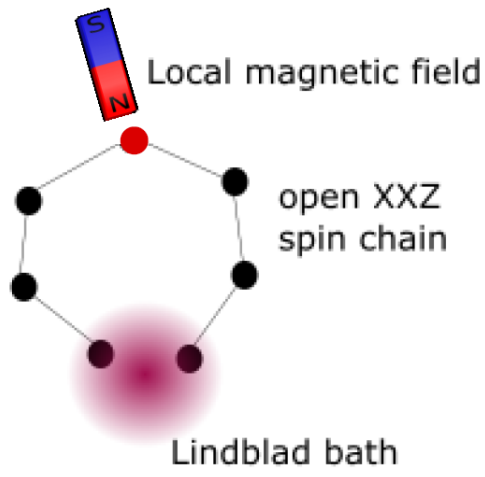

Figure 1: Schematic setup for a quantum switch. A spin chain strongly coupled to a dissipative magnetization bath at the ends is controlled by a local magnetic field applied to a single interior spins of the chain.

dissipation and in the presence of a local magnetic field applied to a single spin in the interior of the chain, while the ends are kept at fixed polarizations by dissipation (see Fig. 1 for a schematic setup). Quite remarkably, we find that suitable changes of the local field permit to switch between pure NESS with opposite chiralities and to invert the spin current while preserving the purity of the states and achieving optimal transport. For possible practical implementations of our theoretical concept, we provide explicit estimates of the relaxation times and fidelities of the NESS involved in the switch for the specific case of a chain of five spins.

We remark that in absence of magnetic fields the optimal transport (maximal magnetization current) in open $X X Z$ chains is always achieved in the proximity of pure states [15] and in the limit of infinitely large dissipative couplings, i.e. the so called Zeno limit [16, 17], it is possible to target pure NESS that carry high ballistic currents and remain pure on a very long time scale $[18,19]$. Dissipative $X X Z$ spin chains have also been recently proposed as sensitive magnonic logical gates [20] as well as quantum diodes for the rectification of the spin [21] and thermal [22,23] currents. However, the possibility to switch different quantum NESS only by means of a local magnetic field is, to the best of our knowledge, a 
completely uninvestigated physical phenomenon of potential interest for applications.

Model. Let us consider the Heisenberg $X X Z$ spin chain of length $N$ coupled to dissipative magnetization baths which tend to polarize the first and last spin of the chain in the states $\psi_{L} \equiv\left|\theta_{L}, \varphi_{L}\right\rangle$ and $\psi_{R} \equiv\left|\theta_{R}, \varphi_{R}\right\rangle$, respectively, along the corresponding polarization vectors: $\vec{n}_{\alpha} \equiv$ $\left(\left\langle\sigma_{i_{\alpha}}^{x}\right\rangle,\left\langle\sigma_{i_{\alpha}}^{y}\right\rangle,\left\langle\sigma_{i_{\alpha}}^{z}\right\rangle\right)$ with $\alpha=L, R$ and $i_{L}=1, i_{R}=N$. Here $\left|\theta_{i}, \varphi_{i}\right\rangle=\left|\cos \frac{\theta_{i}}{2} e^{-i \frac{\varphi_{i}}{2}}, \sin \frac{\theta_{i}}{2} e^{i \frac{\varphi_{i}}{2}}\right\rangle$ denotes the pure single spin state at site $i, \vec{\sigma}$ are the usual Pauli matrices and $\theta_{i}, \varphi_{i}$ are spherical coordinates of the local magnetization, i.e. $\left(\left\langle\sigma_{i}^{x}\right\rangle,\left\langle\sigma_{i}^{y}\right\rangle,\left\langle\sigma_{i}^{z}\right\rangle\right)=\left(\sin \theta_{i} \cos \varphi_{i}, \sin \theta_{i} \sin \varphi_{i}, \cos \theta_{i}\right)$. The interaction with the dissipative environment is described in the framework of the Lindblad master equation (LME) [24, 25]:

$$
\frac{\partial \rho(t)}{\partial t}=\mathcal{L}[\rho(t)]=-\frac{i}{\hbar}\left[H_{0}, \rho(t)\right]+\Gamma \sum_{i=1}^{2} \mathcal{D}_{L_{i}}[\rho(t)],
$$

where $\mathcal{L}$ is the Liouvillean operator generating the full evolution of the state and $H_{0}$ is the $X X Z$ spin chain Hamiltonian with uniaxial anisotropy describing the coherent part of the evolution:

$$
H_{0}=J \sum_{n=1}^{N-1}\left(\sigma_{n}^{x} \sigma_{n+1}^{x}+\sigma_{n}^{y} \sigma_{n+1}^{y}+\Delta\left(\sigma_{n}^{z} \sigma_{n+1}^{z}-I\right)\right) .
$$

In Eq. (1) $\Gamma$ denotes the dissipation strength, $\mathcal{D}_{L} X \equiv$ $L X L^{\dagger}-\frac{1}{2}\left(L^{\dagger} L X+X L^{\dagger} L\right)$, with $L_{i}$ Lindblad operators targeting the polarizations $\vec{n}_{L}, \vec{n}_{R}$ at the left (site 1) and right (site $N$ ) edges, respectively, taken as

$$
L_{1}=\left|\theta_{L}, \varphi_{L}\right\rangle\left\langle\theta_{L},\left.\varphi_{L}\right|^{\perp}, \quad L_{2}=\mid \theta_{R}, \varphi_{R}\right\rangle\left\langle\theta_{R},\left.\varphi_{R}\right|^{\perp},\right.
$$

with $|\theta, \varphi\rangle^{\perp}$ denoting the state orthogonal to $|\theta, \varphi\rangle$. It is a generic feature of Eq. (1) that, independently on initial conditions, after a relaxation time a NESS is reached: $\rho(t) \rightarrow$ $\rho_{N E S S}$. Due to the gradient of magnetization created by the dissipative baths, a generic NESS is intrinsically a nonequilibrium state, unique for our choice of dissipators. By changing the bath polarizations $\vec{n}_{L}, \vec{n}_{R}$ and adjusting the anisotropy parameter, $\Delta$, one can manipulate the NESS to obtain a pure state $\rho=|S H S\rangle\langle S H S|$ [18], [26], with

$$
|S H S\rangle \equiv \prod_{k=0}^{N-1} \otimes|\theta, k \gamma\rangle=\prod_{k=0}^{N-1} \otimes\left(\begin{array}{c}
\cos \left(\frac{\theta}{2}\right) e^{-\frac{i}{2} k \gamma} \\
\sin \left(\frac{\theta}{2}\right) e^{\frac{i}{2} k \gamma}
\end{array}\right)
$$

and $\gamma=\arccos \Delta$. Note that the azimuthal angle in (4) increases by $\gamma$ at each site along the chain so that the spins build up a fully polarized frozen helix of period $2 \pi / \gamma$, also called spin-helix state (SHS). To relax on a SHS the dissipation $\Gamma$ must be much stronger than the coherent part of the evolution and polarization baths must fit the SHS, i.e. $\psi_{L} \equiv|\theta, 0\rangle, \psi_{R} \equiv|\theta, \gamma(N-1)\rangle$. Also note that the relation $\Delta=\cos \gamma$ implies that SHS with opposite chiralities, i.e. $|S H S(\theta, \pm \gamma)\rangle \equiv| \pm\rangle_{1, N}$, can exist for the same value of $\Delta$ and they carry opposite ballistic currents:

$$
{ }_{1, N}\left\langle \pm\left|\hat{j}_{k}^{z}\right| \pm\right\rangle_{1, N}= \pm 2 J \sin \gamma \sin \theta
$$

with $\hat{j}_{k}^{z}=2 J\left(\sigma_{k}^{x} \sigma_{k+1}^{y}-\sigma_{k}^{y} \sigma_{k+1}^{x}\right)$ denoting the $z$-component of the magnetization current operator.

Pure kink-SHS. In order to manipulate the spin-helix NESS by means of local magnetic fields we consider the Hamiltonian $H=H_{0}+\vec{h}_{M} \cdot \vec{\sigma}_{M}$ with $H_{0}$ given by (2) and $\vec{h}_{M}$ denoting a magnetic field applied to a bulk spin at site $M$, $1<M<N$. In the following we assume, for simplicity, boundary bath polarizations in the $X Y$-plane $(\theta=\pi / 2)$ and denote $|\varphi\rangle \equiv|\pi / 2, \varphi\rangle$. The above SHS properties allow to construct a novel type of NESS consisting of two SHS pieces of opposite chirality joined at the site $M$. Indeed, by properly choosing $\vec{h}_{M}$ and the boundary bath polarizations, it is possible to stabilize via dissipation the pure NESS:

$$
\begin{aligned}
|+,-\rangle_{M} & \equiv|+\rangle_{1, M} \otimes|-\rangle_{M+1, N}= \\
= & \prod_{k=0}^{M-1} \otimes|k \gamma\rangle \prod_{k=1}^{N-M} \otimes|(M-1-k) \gamma\rangle
\end{aligned}
$$

that is exact in the limit $\Gamma \rightarrow \infty$ (see Supplementary Material [29]). Obviously, such a state can exist only due to the presence of the magnetic field that sustains the change of chirality (kink) created at the site $M$. Indeed, note that at the site $M$ the state (6) undergoes an inversion of chirality, i.e. $|+,-\rangle_{M} \equiv \prod_{k=1}^{N}\left|\varphi_{k}\right\rangle$ with $\varphi_{k+1}-\varphi_{k}=\gamma$ for $k<M$ and $\varphi_{k+1}-\varphi_{k}=-\gamma$ for $k \geq M$. In the following we refer to this state as a kink-spin helix state (kink-SHS).

The fact that the $|+\rangle$ and $|-\rangle$ pieces of the kink-SHS (6) carry opposite ballistic currents (see Eq. (5)) implies that the site $M$ must be either a source or a sink, depending on the sign of $\gamma$, and in order to have stationarity the following balance condition for $\left\langle\sigma_{M}^{z}\right\rangle$ must be satisfied:

$$
0=\frac{\partial}{\partial t}\left\langle\sigma_{M}^{z}\right\rangle=\left\langle\hat{j}_{M-1}^{z}-\hat{j}_{M}^{z}-i\left[\sum_{\alpha=1}^{3} h_{M}^{\alpha} \sigma_{M}^{\alpha}, \sigma_{M}^{z}\right]\right\rangle .
$$

Calculating the averages in (7) with respect to the kink-SHS (6) and using (5) we find:

$$
h_{M}^{y} \cos (\gamma(M-1))-h_{M}^{x} \sin (\gamma(M-1))=2 J \sin \gamma .
$$

Note that this condition on the magnetic field does not involve the $h_{M}^{z}$ component. A more elaborate analysis (see Supplementary Material [29]) yields that $h_{M}^{z}=0$, together with two other conditions: i) the relation $\Delta=\cos \gamma$ and ii) the compatibility of the state (6) with the boundary polarizations, i.e. $\psi_{L}=\left|\varphi_{L}\right\rangle, \quad \psi_{R}=\left|\varphi_{R}\right\rangle$, with $\varphi_{L}=0$ and $\varphi_{R}=(2 M-N-1) \gamma$.

Condition (8) plays a central role in the following. Indeed, for any given $N, M$, Eq. (8) and the above condition i) define a hyperplane in the parameter space $\left\{\Delta, h_{M}^{x}, h_{M}^{y}\right\}$ where pure kink-SHS exist. A $2 D$ cut, along the $h_{M}^{x}=h_{M}^{y}$ direction of the hyperplane is shown in Fig. 2a. By tuning the local magnetic field components in the $X Y$ plane according to Eq. (8) and increasing the dissipation rate $\Gamma$, the NESS can be made arbitrarily close to the pure NESS (6). This is shown in Fig. $2 b$ where the magnetization current and $1-p$, with 


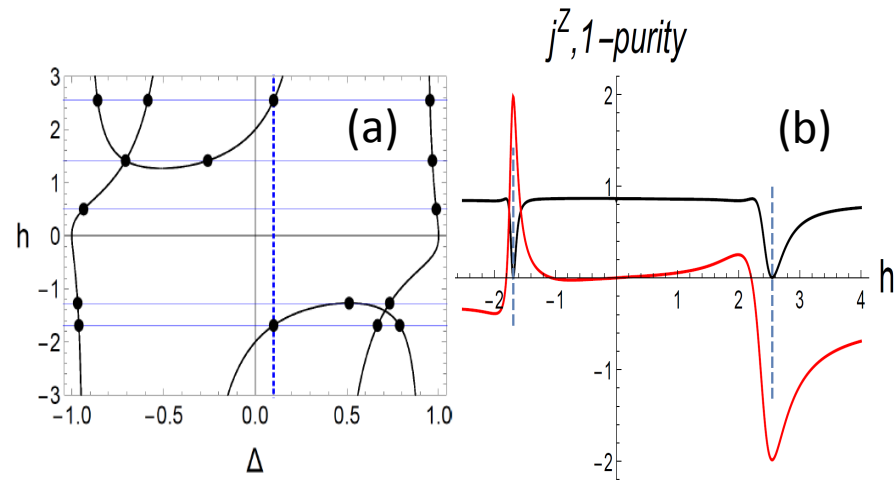

Figure 2: Panel (a). Kink SHS existence region (thick continuous curves) in the parameter space $(\Delta, h)$ as obtained from Eq. (8) for a spin chain of size $N=5$ with $J=1$ and magnetic field $h\left(\sigma_{3}^{x}+\sigma_{3}^{y}\right)$ applied at site $M=3$. Note the symmetry of the curves under the transformation $h(\Delta) \rightarrow-h(-\Delta)$. Dots refer to kink-SHS obtained from numerical solutions of the stationary LME for $\Gamma=10^{3}$ and magnetic field amplitudes corresponding to the depicted horizontal thin lines $h=2.54797,1.414215,0.5,-1.267582,-1.688785$. Panel (b). Rescaled purity, $1-p$, (black curve) and steady magnetization current measured at the link between sites 1 and 2 of the chain (red curve) obtained from numerical solutions of the stationary LME as functions of the magnetic field amplitude $h$. Parameter values are the same as in panel (a) but $\Delta=0.1, \varphi_{L}=\varphi_{R}=0$. Dashed lines show the location of the pure kink-SHS $|+,-\rangle$ and $|-,+\rangle$, which are exact mirror brothers predicted by (8) for $h_{M}^{x}=h_{M}^{y} \equiv h$, $\gamma= \pm \arccos \Delta$, and corresponding to the bottom and top dots on the vertical dotted line $\Delta=0.1$ of the left panel, respectively.

$p=\operatorname{tr}\left(\rho_{N E S S}^{2}\right)$ denoting the purity, are reported as functions of the magnetic field amplitude $h$, for the same spin chain and parameter values considered in Fig. 2a, but for $\Delta=0.1$ and $\varphi_{L}=\varphi_{R}=0$. From this we see that $1-p=0$ (i.e. kink-SHS are pure states) exactly in correspondence of the predicted values of $h$ (compare with $h$ values of points on the vertical dashed line in Fig. 2a). Moreover, the current at these points attains the same maximum amplitude but with opposite signs, as expected for opposite chirality, $|+,-\rangle$, $|-,+\rangle$ kink-SHS. Also note that since the kink-SHS condition $\Delta=\cos \gamma$ couples the anisotropy to the boundary magnetization gradient $\varphi_{R}-\varphi_{L}$, a change of the anisotropy or a change of the chirality (i.e. $\gamma \rightarrow-\gamma$ ), makes necessary an adjustment of the magnetization gradient. If $N$ is odd and the field is applied at the center of the chain, however, the constraint $\varphi_{R}=(2 M-N-1) \gamma$ is satisfied for aligned borders, $\varphi_{L}=\varphi_{R}=0$, for arbitrary $\gamma$.

A kink-SHS quantum switch. Let us consider a chain of odd size $N$ with a local magnetic field applied to the central site, $M=(N+1) / 2$ and $\varphi_{R}=\varphi_{L}=0$. In this case the bath reservoirs are the same for both edges (as sketched in Fig. 1)) and Eq. (8) is written as:

$$
h_{M}^{y} \cos \frac{(N-1) \gamma}{2} \mp h_{M}^{x} \sin \frac{(N-1) \gamma}{2}= \pm 2 J \sin \gamma,
$$

where the different signs correspond to the kink-SHS $|+,-\rangle$ and $|-,+\rangle$ obtained from Eq. (6) with $+\gamma$ and $-\gamma$, respectively.

From Eq. (9) it follows that, by keeping the magnetic field component amplitude $h_{M}^{y}$ fixed and flipping its sign, one

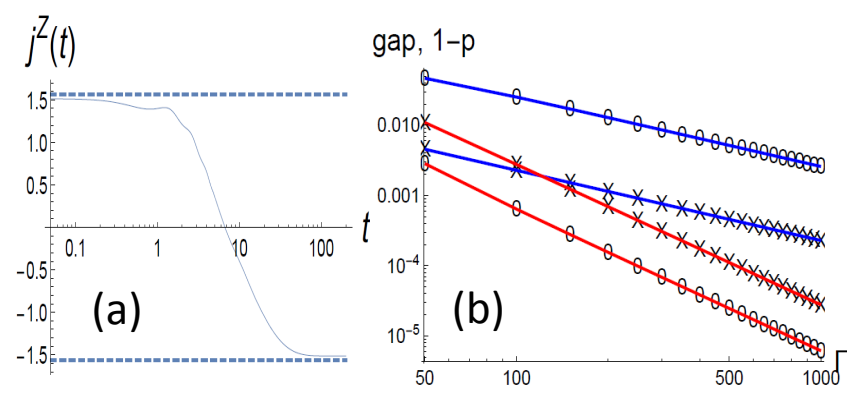

Figure 3: Panel (a).Time evolution of the magnetization current $j^{z}$ measured at the link between sites 1,2 after an abrupt change of a magnetic field, $\left(h_{M}^{x}, h_{M}^{y}, 0\right) \rightarrow\left(h_{M}^{x},-h_{M}^{y}, 0\right)$ at time $t=0$, applied to the middle site. Initial condition is a right-handed kink-SHS (the NESS for $h_{M}^{y}<0$ ), final state is the left handed kink-SHS (the NESS for $h_{M}^{y}>0$ ). Parameters values are $J=1, N=5, M=3$, $\Gamma=20, \Delta=\cos 2 \pi / 7, \varphi_{L}=\varphi_{R}=0$. Local field amplitudes $h_{M}^{x}=-1.775, h_{M}^{y}=0.75$, correspond to the optimal choice indicated by the dotted line in Fig. 4 and satisfy the constraint (9) with "+ "sign. Dashed lines show the magnetization currents $\left\langle j^{z}\right\rangle=2 \sin (2 \pi / 7)$ of the asymptotic Zeno states. Panel (b). Numerical data for the Liouvillean gap (upper data points joined by blue lines) and for $1-\operatorname{tr} \rho^{2}$ (lower data points joined by red lines) of the NESS as function of $\Gamma$, illustrating the scaling properties in (10). Parameter values are fixed as in panel (a) except for markers " $\times$ " for which $h_{M}^{x}=-2.0603, h_{M}^{y}=2.0$. Blue and red lines with slopes $1 / x$ and $1 / x^{2}$, respectively, are drawn to guide the eyes.

can pass from the pure state $|+,-\rangle$ to the pure state $|-,+\rangle$ (or viceversa), realizing in this manner a quantum dissipative switch that operates only via the local magnetic field. This is illustrated in Fig. 3a where the time evolution of the magnetization current $j^{z}$, obtained from direct numerical integrations of the LME (1), is shown after an instantaneous change of the magnetic field. In the numerical simulation the initial condition is taken as a kink-SHS of type $|+,-\rangle$, created by tuning the local field $\left(h_{M}^{x}, h_{M}^{y}, 0\right)$ to the corresponding anisotropy value using Eq. (9), and by flipping, at the time $t=0$, the $y$-component of the field. The net outcome of the flip is the change of the initial state $|+,-\rangle$ into the kink-SHS $|-,+\rangle$ of opposite chirality, this giving the inversion of the current shown in Fig. 3a.

Fidelity and relaxation time optimization. Although exact pure kink-SHS are attained for infinitely large dissipations, for any practical purpose it is possible to achieve an effective Zeno regime when the dissipation becomes larger than some characteristic finite value, $\Gamma_{\text {char }}$, which depends on system parameters. For finite $\Gamma$ the distance of the NESS from the asymptotic Zeno pure state can be measured with the purity $p$ while the relaxation time, $\tau$, i.e. the time needed to reach the asymptotic state, is of the order $\tau \approx 1 / g$ where $g$ is the gap of the Liouvillean superoperator associated to the LME (1). In Fig. 3b we show the Liouvillean gap and the purity obtained from direct numerical solutions of the LME, as functions of $\Gamma$, from which we see that:

$$
1-p=\frac{\Gamma_{\text {char }}^{2}}{\Gamma^{2}}+o\left(\Gamma^{-2}\right), \quad g=\frac{D}{\Gamma}+o\left(\Gamma^{-1}\right),
$$




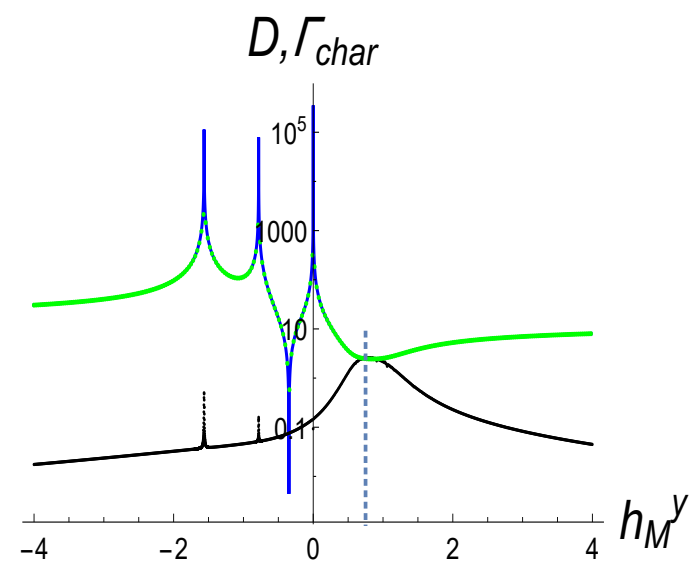

Figure 4: $\Gamma_{\text {char }}$ (upper dotted green line) and $D$ (lower dotted black line) versus $h_{M}^{y}$ as obtained from direct numerical calculations of Eq. (1) for parameter values fixed as in Fig. 3a but with $\Gamma=3000$. The continuous blue line refers to exact analytical expression of $\Gamma_{\text {char }}$ derived in Eq. (9) of the Supplementary Material [29]. The optimal choice of $h_{M}^{y} \approx 0.75$ is indicated by the dotted vertical line.

with $\Gamma_{\text {char }}, D$, depending on system parameters but not on $\Gamma$. From this it follows that for a better and faster convergence to the Zeno pure NESS one must minimize $\Gamma_{\text {char }}$ and maximize $D$. This can be done by observing that Eq. (9) implies the existence of a one-parameter family of solutions for $h_{M}^{x}, h_{M}^{y}$ which corresponds exactly the the same kink-SHS, this allowing to choose one of the components of the magnetic field freely. Taking the $y$-component $h_{M}^{y}$ as a free parameter, we have investigated in Fig. 4 how $D$ and $\Gamma_{\text {char }}$ depend on it. We see that the dependence on $h_{M}^{y}$ is huge and, for the considered case, the optimal choice is obtained in the interval $h_{M}^{y} \approx 0.75 \pm 0.25$, where $D$ and $\Gamma_{\text {char }}$ reach their max and min, respectively (note that $D$ and $\Gamma_{\text {char }}$ may vary by orders of magnitude outside this interval). Moreover, for opposite chirality $\pm \gamma_{0}$ states we find

$$
\begin{aligned}
D\left(h_{M}^{y},+\gamma_{0}\right) & =D\left(-h_{M}^{y},-\gamma_{0}\right), \\
\Gamma_{\text {char }}\left(h_{M}^{y},+\gamma_{0}\right) & =\Gamma_{\text {char }}\left(-h_{M}^{y},-\gamma_{0}\right),
\end{aligned}
$$

these being a consequence of the mirror symmetry of the respective physical states and imply that the $D, \Gamma_{\text {char }}$ optimization holds true for both $\left( \pm h_{M}^{y}, \pm \gamma_{0}\right)$ parameter choices, i.e. for both kink-SHS involved in the switch.

Conclusion. We have demonstrated the possibility to use local magnetic fields to switch between different pure NESS, preserving their purity and attaining optimal spin current. A quantum switch that inverts the current via the local field only, was discussed for the case of "aligned borders". The developed theory, however, is general and results can be extended to arbitrary bath polarizations. The optimization of the relaxation time and fidelity of the NESS involved in the switch was also discussed. We believe that the above properties and the robustness of kink-SHS against decoherence makes these NESS of interest for applications.

Acknowledgements. V.P. acknowledges support from the
European Research Council (ERC) through the advanced grant 694544 - OMNES and from the DFG grant KL 645/20-1. M.S. acknowledges support from the Ministero dell'Istruzione, dell'Universitá e della Ricerca (MIUR) through the grant PRIN-2015-K7KK8L on "Statistical Mechanics and Complexity".

[1] M. A. Nielsen and I. L. Chang, Quantum computation and Quantum Information, Cambridge University Press, 2000.

[2] J. Marshall, L. Campos Venuti, and P. Zanardi, Phys. Rev. A 94 (2016) 052339.

[3] Y. Ikeda and N. Yamamoto, Phys. Rev. A 87 (2013) 033802.

[4] C. Cormick, A. Bermudez, S.F. Huelga, and M. B. Plenio, New J. Phys. 15 (2013) 073027.

[5] E.G. Dalla Torre, J. Otterbach, E. Demler, V.Vuletic, and M.D. Lukin, Phys. Rev. Lett. 110 (2013) 120402.

[6] Y. Lin et al., Nature 504 (2013) 415.

[7] Z. Leghtas, U. Vool, S. Shankar, M. Hatridge, S. M. Girvin, M. H. Devoret, and M. Mirrahimi, Phys. Rev. A 88 (2013) 023849.

[8] W. Yi, S. Diehl, A.J. Daley, and P. Zoller, New J. Phys. 14 (2012) 055002.

[9] K. Stannigel, P. Rabl and P. Zoller, New J. Phys. 14 (2012) 063014.

[10] F. Ticozzi, L. Viola, Phil. Trans. R. Soc. A 3705259 (2012).

[11] H. Krauter, C.A. Muschik, K. Jensen, W. Wasilewski, J.M. Petersen, J.I. Cirac, and E.S. Polzik, Phys. Rev. Lett. 107 (2011) 080503.

[12] Yasushi Kondo, Yuichiro Matsuzaki, Kei Matsushima, and Jefferson G Filgueiras, New J. Phys. 13 (2016) 013033.

[13] S. Diehl, A. Micheli, A. Kantian, B. Kraus, H. P. Büchler, and P. Zoller, Nat. Phys. 4 (2008) 878.

[14] F. Verstraete, M.M. Wolf, and J.I. Cirac, Nature Physics 5 (2009) 633.

[15] M. Salerno, V. Popkov, Phys. Rev. E 87 (2013) 022108.

[16] B. Misra and E.C.G. Sudarshan, J. Math. Phys. 18 (1977) 756.

[17] K. Koshino and A. Shimizu, Phys. Rep. 412,(2005) 191.

[18] V. Popkov, C. Presilla, Phys. Rev. A 93 (2016) 022111.

[19] V. Popkov, C. Presilla, and J. Schmidt, Phys. Rev. A 95 (2017) 052131.

[20] G.T. Landi, D. Karevski, Phys. Rev. B 91 (2015) 174422 (2015).

[21] V. Balachandran, G. Benenti, E. Pereira, G. Casati, and D. Poletti, Phys. Rev. Lett. 120, 200603 (2018).

[22] G.T. Landi, E. Novais, M.J. de Oliveira, D. Karevski, Phys. Rev. E 90 (2014) 042142.

[23] V. Balachandran, G. Benenti, E. Pereira, G. Casati, and D. Poletti, Phys. Rev. E 99, 032136 (2019).

[24] H.P. Breuer and F. Petruccione, The Theory of Open Quantum Systems, Oxford University Press, (2002).

[25] S.R. Clark, J. Prior, M.J. Hartmann, D. Jaksch and M. B. Plenio, New J. Phys. 12 (2010) 025005.

[26] V. Popkov, J. Schmidt, and C. Presilla, J. Phys. A: Math. Theor. 50 (2017) 435302.

[27] V. Popkov, S. Essink, C. Presilla, G. M. Schütz, Phys. Rev. A 98 (2018) 052110.

[28] P. Zanardi and L. Campos Venuti, Phys. Rev. Lett. 113 (2014) 240406.

[29] See the Supplementary Material for the proof of the Zeno kinkSHS purity and for an analytical derivation of $\Gamma_{\text {char }}$. 